\title{
A driving simulator study on the perception of distances in situations of car-following and overtaking
}

\author{
J. Bergeron $^{1}$, B. Baumberger ${ }^{2}$, M. Paquette ${ }^{1}$, M. Flückiger ${ }^{2}$ \\ \& A. Delorme ${ }^{1}$ \\ ${ }^{1}$ University of Montreal, Canada \\ ${ }^{2}$ University of Geneva, Switzerland
}

\begin{abstract}
The principal objective of this experiment was to test how a subject can control his approach towards several simulated car-targets in different contexts of carfollowing and overtaking. We assume that these complex situations may influence driving performance according to the difficulty in perceiving distances properly. A second objective was to evaluate the influence of driving experience in the perception of distances in these situations. Fourteen drivers $(7$ men and 7 women) between 20 and 56 years old participated to this study. Their driving experience ranged between 2.5 and 32 years and they drove between 1000 and $25000 \mathrm{~km}$ per year The subjects' first task consisted in stopping their car at equal distance between two static cars separated either by 34.5 or $54.5 \mathrm{~m}$. The second and third tasks were typical manoeuvres of overtaking, subjects having to place their car at equal distance between two preceding cars running at 40 or $60 \mathrm{~km} / \mathrm{h}$. Results show that the subjects underestimated distances in any conditions. They were better at adjusting their own position at $60 \mathrm{~km} / \mathrm{h}$ than at 40 $\mathrm{km} / \mathrm{h}$, and their performance increased with smaller car distances. The participants' driving experience (number of years) was the most important individual variable in the differentiation of subjects' performances. These results may be interpreted in terms of the importance of driving experience in situations of car-following and overtaking, in which drivers must estimate the position of other vehicles in reference to their own vehicle.
\end{abstract}

Keywords: driving simulator, distance perception, car-following, overtaking, driving experience. 


\section{Introduction}

Car-following and overtaking performances are essential parts of car driving. In these situations, evaluation of vehicle speed and inter-vehicle distance is crucial skills and constant demands [1]. Ability of drivers to properly perceive the distance and scale the relative velocity between their own and a lead vehicle is critical in car-following. In manoeuvres of overtaking, estimating distances is more complex. Drivers must take into account the distances of the different vehicles in front of them as well as that of vehicles coming from the opposite direction and possibly the distance of vehicles behind them, prior to making a decision to overtake. In such situations drivers need to estimate the position and the speed of other drivers in addition to their own position and velocity (Baumberger et al. [2]). The principal objective of the resent study was to test how a subject can control his approach towards several simulated car-targets in different contexts of car-following and overtaking.

Moreover, accurately positioning a vehicle in space not only requires estimating the distance, but also memorising this estimation and transposing it according to the information available at the time of the final estimation. From a perceptual point of view, in such situations the drivers' task consists in estimating the relative position of objects. After the correct position has disappeared from their visual field, drivers must then estimate their own position relative to the objects present in the visual environment without any visual feedback control.

Most of the experiments on these issues are performed by simulation in the laboratory, because of the difficulty and danger of performing such studies on the road (Hoffmann and Mortimer [3]). The difficulty arises from setting up relative speeds accurately; the danger from the short periods of time-to-collision in some of the experimental conditions (Cavallo and Laurent [4]). The use of such a simulation may not be a problem, despite limitations with regard to the role of peripheral vision since it is largely the visual information related to the changes in subtended angle that is used for such estimations. When both vehicles are in motion, like in car-following and overtaking, the useful visual information is largely gained from the central visual field of the following driver [3].

Important inter-individual differences have been found in many investigations bearing on drivers' performance on visual perception and ability to estimate the relative position of objects in the visual field [3, 5-8]. Driving experience may be suspected to be one of the most significant factors explaining these intraindividual differences. A second objective of our study was to evaluate the influence of driving experience in the perception of distances in the situations of car-following and overtaking. 


\section{Method}

\subsection{The University of Montreal driving simulator}

\subsubsection{Hardware}

The driving simulator of the University of Montreal is a fixed-base simulator composed of a full size vehicle cab (Honda Civic), fully functional control pedals, steering wheel and dashboard, projected LCD speedometer/tachometer cluster, and torque motor developed to provide realistic force feedback. These components are interactive with various road scenes projected on a large screen (about $3 \mathrm{~m}$ by $2.5 \mathrm{~m}$ ) and intended to make steering, braking, and accelerating look and feel as though one were actually driving. Traffic is programmable, either following the general rules of the road or as scripted. Many simulated worlds are created, such as scenes from rural, urban and expressway settings, including intersections with traffic signals. Simulated highways have been designed using actual Canadian geometric route design standards. The vehicle dynamics can be changed.

Driver and vehicle performance (steering wheel angle, speed, lane position, etc.) are recorded at up to $50 \mathrm{~Hz}$ by the main simulator computer, and performance on in-cab tasks is recorded by other IBM - compatible computers. In addition, drivers' actions (their face, hands, the instrument panel feet and foot controls) can be recorded by a video system.

\subsubsection{Software}

The software has been conceived as to create realistic traffic environments that can include other vehicles, traffic lights and signs, and a wide range of street and highway configurations, including intersections and other roadway geometries. Buildings can be efficiently represented by only those surfaces that would be visible to the subject while operating on an adjacent roadway. The basic library of objects with the visual database modeling software includes passenger vehicles, trucks and delivery vans. These basic objects are modifiable using the software as to color and some minor physical arrangements to produce vehicles that visually differ from each other.

\subsubsection{Measures of drivers' performance}

During a simulation test, numerous data are registered including the location and speed of the vehicle on the $\mathrm{X}, \mathrm{Y}$ and $\mathrm{Z}$ axes, number of times the vehicle goes beyond the road borders, and number of collisions. Critical events can be introduced, and the driver's responses (e.g., braking, steering changes) can be measured. Different kinds of collision events are possible: loss of control and running off the road, collision with fixed objects on the road (e.g. a pothole in the pavement), and collision with a fixed object along the road (e.g. a traffic signal, a tree, a house, other vehicles stalled on the road or parked on the roadside).

\subsection{Subjects of the present study}

The participants were fourteen drivers ( 7 men and 7 women) between 20 and 56 years old (mean 27 years $+/-9.6$ years). Their driving experience ranged 
between 2.5 and 32 years (mean 9 years $+/-8$ years) and they drove between 1 000 and $25000 \mathrm{~km}$ per year (mean $13000 \mathrm{~km}+/-7800 \mathrm{~km}$ ). First, they were introduced to the simulator and given a demonstration of the controls. The subjects' first task consisted in stopping their car at equal distance between two static cars separated either by 34.5 or $54.5 \mathrm{~m}$. The second and third tasks were typical manoeuvres of overtaking, subjects having to place their car at equal distance between two preceding cars running at 40 or $60 \mathrm{~km} / \mathrm{h}$, separated either by 34.5 or $54.5 \mathrm{~m}$.

\subsection{Procedure}

In the present study, the virtual environment was composed of a straight road lying ahead of the car. The surrounding scenery was covered with grass, bushes and trees. Drivers could actively change the speed of their vehicle, which was moving in the left lane of the simulated road. To decrease the difficulty of the driving task and to increase the quality of the distances estimation, the subjects could not change direction and could only break or accelerate. Two reference vehicles (B and $\mathrm{C}$ ) were positioned in the right lane (see figure 1) at a distance of $40 \mathrm{~m}$ or $60 \mathrm{~m}$ (Distance factor: respectively 34.5 and $54.5 \mathrm{~m}$ between the rear end of $C$ and the front of B). These vehicles were either motionless $(0 \mathrm{~km} / \mathrm{h})$ or in motion at $40 \mathrm{~km} / \mathrm{h}$ or $60 \mathrm{~km} / \mathrm{h}$ (Speed factor). The task required of the drivers was to position the front of the car they were driving (A) according to the position of vehicles (B and $\mathrm{C}$ ) in three situations of distance estimation.

The subject was free to ask any questions about the simulator or the tasks before the beginning of the experimental session. When he or she was judged to be able to perform all tasks without any supervision, the experimental trials could begin. Each of the three situations of distance estimations was reproduced three times in one session.

\section{Results}

An analysis of variance for repeated measures (anova) was performed with three intra-subject factors: trials (three situations of distance estimations); speed of displacement (speed factor: 0,40 and $60 \mathrm{~km} / \mathrm{h}$ ) and distance between vehicles (distance factor: respectively 34.5 and $54.5 \mathrm{~m}$ ). The analysis was carried out on the positioning error of the subject-drivers' car. A positive answer corresponds to an overestimation, meaning that the answer has overshot the mid-distance between target-cars B and C. Conversely, a negative answer corresponds to an underestimation, meaning that the answer given was provided before the correct position.

The results show that subjects under-estimated distances. Performances were significantly better $(F(2,22)=26.07, p<.01)$ in the static target condition $(0 \mathrm{~km} / \mathrm{h})$ than in the two moving target conditions. Also, subjects were more accurate when they were moving at $60 \mathrm{~km} / \mathrm{h}$ than $40 \mathrm{~km} / \mathrm{h}(\mathrm{F}(1,12)=8.25, \mathrm{p}<.014)$. Finally, subjects' estimations were more accurate $(F(1,11)=16.17, p<.01)$ when the distance between the vehicles was smaller $(34.5 \mathrm{~m})$. 


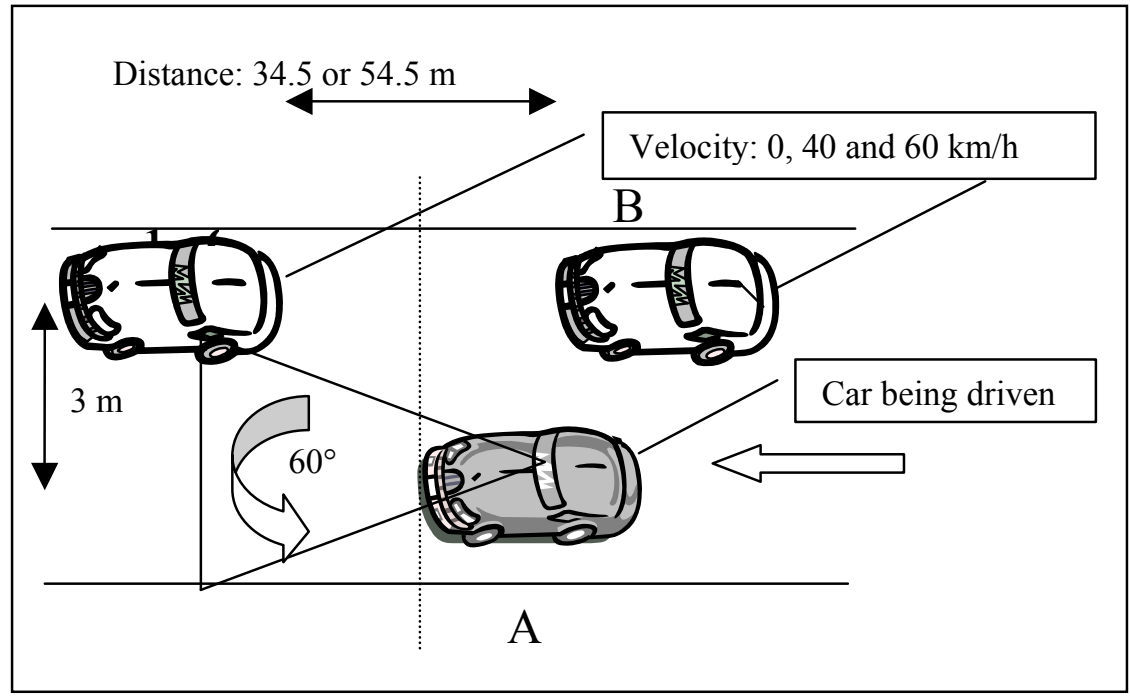

Figure 1: Illustration of the experimental condition.

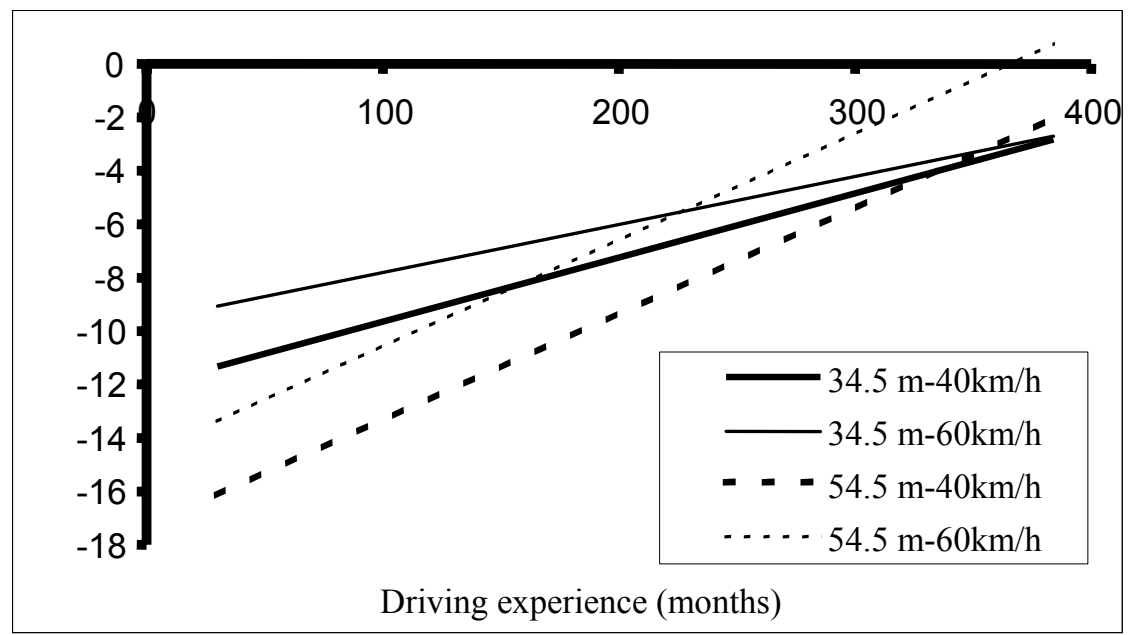

Figure 2: Relations between estimation of distance and driving experience.

A post-hoc analysis of variance (anova) on the positioning error relative to the distance between vehicles (Webber ratio) doesn't show any significant effect according to the distance between the vehicles. Thus localization error is only influenced by the speed (speed: 0,40 and $60 \mathrm{~km} / \mathrm{h} \mathrm{F}(2,22)=13.6, \mathrm{p}<.001)$ and the pairwise comparison between $0-40 \mathrm{~km} / \mathrm{h} \quad(\mathrm{t}(10)=4.42, \mathrm{p}<.001,0-60 \mathrm{~km} / \mathrm{h}$, $\mathrm{t}(10)=2.48, \mathrm{p}<.032)$ and $40-60 \mathrm{~km} / \mathrm{h}(\mathrm{t}(10)=2.87, \mathrm{p}<.015)$; it corresponds respectively to an error of $12.1,22.5$ and $18 \%$ of the distance between vehicles $\mathrm{B}$ and $\mathrm{C}$. 
The participants' driving experience (number of months since the subject have a driving licence) was revealed as an important individual variable in the differentiation of subjects' performances, as shown in Figure 2.

\section{Discussion}

Results show that the subjects underestimated distances in any conditions. They were better at adjusting their own position at $60 \mathrm{~km} / \mathrm{h}$ than at $40 \mathrm{~km} / \mathrm{h}$, and their performance increased with smaller car distances.

Our hypothesis concerning drivers' poorer performance when required to position themselves relative to other moving vehicles was confirmed. In the nonmoving condition, performances were systematically better than in the moving conditions, positioning oneself between two vehicles involves controlling many more parameters and represents an increase in constraints related to the type of task that has a direct impact upon the quality of the estimations of distance. Moreover, our results showed that subjects' performance improved with an increase in speed and with a decrease in distance between the vehicles. Maybe drivers believe that the slower speed and the greater the distance, the less they need to be accurate. More research is needed on this issue.

Our results show also important inter-individual differences between participants, in conformity of numerous experiments bearing on drivers' performance on visual perception and ability to estimate the relative position of objects in the visual field. The participants' driving experience (number of years) was the most important individual variable in the differentiation of subjects' performances.

\section{Conclusion}

These results may be interpreted in terms of the importance of driving experience in situations of car-following and overtaking, in which drivers must estimate the position of other vehicles in reference to their own vehicle.

\section{References}

[1] Kemeny, A. \& Panerai, F., Evaluating perception in driving simulation experiments. Trends in Cognitive Sciences, 7(1), pp. 31-37, 2003.

[2] Baumberger, B., Fluckiger, M., Paquette, M., Bergeron, J., \& Delorme, A, Perception of relative distance in a driving simulator. Japanese Journal of Psychology (in press).

[3] Hoffmann, E.R. \& Mortimer, R.G., Scaling of relative velocity between Vehicles. Accident, Analysis and Prevention, 28(4), pp. 415-421, 1996.

[4] Cavallo, V. \& Laurent, M., Visual information and skill level in time-tocollision estimation. Perception, 17(5), pp. 623-632, 1988. 
[5] Crundall, D.E. \& Underwood, G., Effects of experience and processing demands on visual information acquisition in drivers. Ergonomics, 41(4), pp. 448-458, 1998.

[6] Crundall, D.E., Underwood, G. \& Chapman, P. Selective searching while driving: the role of experience in hazard detection and general surveillance. Ergonomics, 45(1), pp. 1-12, 1999.

[7] Lansdown, T.C., Individual differences during driver secondary task performance: verbal protocol and visual allocation findings. Accident, Analysis and Prevention, 34(5), pp. 655-662, 1999.

[8] Park, K.S., Lee, A.J., \& Koh, B.K., Drivers' Characteristics in the Perception of a Lead Vehicle's Deceleration Level. International Journal of Cognitive Ergonomics, 5(2), pp. 125-136, 2001. 